Revista de Estudios Histórico-Jurídicos

[Sección materiales]

XLIII (Valparaíso, Chile, 2021)

[pp. 835-851]

\title{
La pieza final del puZzLE: Examen De Derecho Natural de don Diego Portales Palazuelos en el Instituto NaCIONAL (18 DE ABRIL DE 1814)
}

[The final piece of the puzzle: examination of the natural law by Diego

Portales Palazuelos at the Instituto Nacional (18 april 1814)]

\author{
René LARROUCAU TORO* \\ Universidad de Chile
}

\section{RESUMEN}

En el contexto de un rastreo de antecedentes organizado por el equipo de ayudantes del profesor Bernardino Bravo Lira, grupo respecto del cual el autor se acababa de incorporar como colaborador, un momento de curiosidad conllevó al descubrimiento de una pieza histórica que, sin saberlo en ese minuto, ayudaría después a complementar una investigación histórico-jurídica realizada mucho antes por el profesor Alejandro Guzmán Brito. Esta era, nada más y nada menos, que el acta del examen que el futuro ministro Diego Portales rindiese sobre materias de Derecho Natural en el naciente Instituto Nacional, en el año 1814. Este documento pone en contexto esta pieza y, a su vez, la pone en relación con los trabajos desarrollados previamente para su apropiado complemento.

\section{Palabras clave}

Derecho Natural - Instituto Nacional - Diego Portales - Heineccius - Historia del Derecho.

\section{Abstract}

In the context of a background trace organized by the team of assistants of Professor Bernardino Bravo Lira, a group for which the author had just joined as a collaborator, a moment of curiosity led to the discovery of a historical piece that, unknowingly at that minute, would then help complement a historical-legal research conducted much earlier by Professor Alejandro Guzmán Brito. This was, no less and nothing less, than the minutes of the review given for the future minister Diego Portales in matters of Natural Law at the nascent National Institute, in 1814. This document puts this piece into context and, in turn, puts it in relationship with the predeveloped works for proper complement.

KEY WORDS

Natural Law - Instituto Nacional - Diego Portales - Heineccius - History of Law.

RECIBIDO el 30 de abril de 2021 y ACEPTADO el 18 de junio de 2021

* Instructor de Historia del Derecho, del Departamento de Ciencias del Derecho, de la Facultad de Ciencias Jurídicas y Sociales de la Universidad de Chile. Dirección postal: Avenida Santa María 076, oficina 201, Providencia, Santiago. Dirección electrónica: rlarroucau@derecho. uchile.cl. ORCID: 0000-0002-7983-2402 
Hace una década atrás, siendo estudiante de Segundo Año, iniciaba mis labores como colaborador de la Cátedra de Historia del Derecho del profesor Bernardino Bravo Lira, en la Facultad de Derecho de la Universidad de Chile. Dentro de mi inexperiencia inevitable, una de las primeras labores que se me asignó fue colaborar con el equipo de ayudantes titulares (entonces conformado por Claudio Barahona, Roberto Cerón y Felipe Peroti) en la búsqueda de antecedentes remitidos a conformar las prosopografías de los distintos miembros de la Judicatura, en los Anales que el profesor Bravo Lira sacaría a la luz durante el año 2011. En ese escenario, me tocó asistir, siendo abril de 2011, a una revisión efectuada dentro de los fondos de la Biblioteca del Instituto Nacional "General José Miguel Carrera Verdugo”. En ese contexto, la curiosidad me llevó a los primeros libros de exámenes, largamente cubiertos de polvo, encontrando una prueba documental que en ese momento no sopesé más allá de la importancia del personaje en particular: la constancia de rendición de exámenes de Derecho Natural por parte de un joven Diego Portales Palazuelos el día 18 de abril de 1814. Fue como encontrar la pieza-esquina de un puzzle, del cual no se alcanza a dimensionar su total magnitud.

Motivado por la curiosidad, fotografié (con la escasa calidad que permitía la cámara de un celular Nokia del año 2009) y transcribí el contenido de esta en una segunda visita, el 21 de abril de 2011 (tengo registro de esta fecha por un correo que enviara al profesor Cristian Román Cordero dándole a conocer lo ya expuesto). Poco tiempo después, tuve la oportunidad de adquirir Portales y el Derecho, obra del profesor Alejandro Guzmán Brito, respecto de la cual su acápite "Las ideas jurídicas de Portales", me permitiera entender el estado de la cuestión sobre el documento: no había registro de su egreso a causa de los convulsos hechos que dieron cierre a la Patria Vieja y que remitía a Benjamín Vicuña Mackenna la referencia que, en efecto, habría alcanzado a dar sus exámenes. Sin embargo, el ilustre tribuno solo dejaba constancia de esta circunstancia y de las materias rendidas (derecho natural y filosofía), así como de su permanencia hasta la clausura del Instituto ${ }^{2}$. Puse en su minuto este antecedente en conocimiento del profesor Guzmán Brito con razón del Congreso de la Sociedad Chilena de Historia del Derecho y Derecho Romano realizado en octubre de ese mismo año en la Universidad Gabriela Mistral, recibiendo su estímulo para que escribiese sobre ello. Guardo agradecido su autógrafo en ese sentido.

Sin embargo, los años pasaron y un pregrado convulso por los tiempos que vivimos, sumado a las permanentes cargas cotidianas, hizo que este proyecto

${ }^{1}$ Guzmán Brito, Alejandro, Las ideas jurídicas de Portales en Boletin de la Academia Chilena de la Historia, 49/93 (1982), pp. 13-42; ahora, en ÉL MISMO, Portales y el derecho (Santiago, 1988), pp. 12 ss. Este fue el discurso de recepción que diera el profesor Guzmán Brito en su ingreso como Académico de Número en la Academia Chilena de la Historia, expuesto con fecha 15 de abril de 1982.

${ }^{2}$ Vicuña Mackenna, Benjamín, Introducción a la bistoria de los diez años de la administración Montt. Don Diego Portales (1974), p. 24. 
se fuera archivando reiteradamente. En el intertanto, más y más fuentes me demostraron que este punto específico de la biografía de Portales no había sido documentado cabalmente. Incluso, por un momento pensé que había perdido definitivamente la referencia. Pero en el estado actual de cosas, bien corresponde saldar esta deuda: primero, con el profesor Guzmán Brito, y seguidamente, conmigo mismo. Por lo mismo, este trabajo no tiene pretensión alguna más que de ser meramente el complemento a lo ya previamente investigado, que permita dar su forma definitiva a este puzle.

\section{Las demás Piezas: los estudios de don Diego Portales previos al INSTITUTO NACIONAL}

Los pasos previos seguidos por don Diego Portales al documento que justifica esta exposición ya se encuentran conocidos y descritos en detalle, por lo cual esta parte del trabajo será meramente una recopilación sintética de ello. Existe cierto consenso relativo sobre que su padre, el superintendente de la Casa de Moneda, don José Santiago Portales, había destinado para él la carrera de la abogacía: esta voluntad será, finalmente, la que condicionará sus estudios hasta el momento en que estos queden truncos definitivamente, en algún momento del año $1815^{3}$.

Sus primeros estudios habrían sido posteriores a 1805: en específico, lecciones de humanidades, en el aula de mayores del latinista don José María Luján ${ }^{4}$. Esta, a juicio de los historiadores, es la época más fructífera de Portales en sus estudios, puesto que de este tiempo heredará un dominio notorio del latín como de ciertos trasuntos de teología.

Siendo el año 1808, ingresó a los 14 años al Real Colegio de San Carlos o Real Convictorio Carolino de Nobles, en la ciudad de Santiago, por medio de la denominada b e c a L e c a r o s ${ }^{5}$. En este lugar debió haber seguido cursos de gramática latina, retórica y filosofía únicamente, ya que la historiografía es conteste en señalar que en esta instancia Portales no habría seguido el curso de cánones y leyes aquí disponible (el cual tenía el carácter de optativo) y que de hecho el texto que habría servido de base a la enseñanza jurídica en este establecimiento

${ }^{3}$ Tanto Vicuña Mackenna, Benjamín, cit. (n. 2), p. 21, como Encina, Francisco Antonio. Portales. Introducción a la historia de la época de don Diego Portales (Santiago, 1934), I, p. 137, señala que en verdad la carrera destinada a Portales era la eclesiástica, "a fin de que gozara de la renta de capellán de la Casa de Moneda".

${ }^{4}$ Sobre Luján, es sabido que entabló oposición a don Juan Egaña por la cátedra de Latinidad y Retórica en la Real Universidad de San Felipe, creada el 10 de junio de 1802, haciendo público "su mérito de maestro público de latinidad y retórica"; al ser confirmado el primero, fue recomendado por el claustro igualmente. MedinA, José Toribio, Historia de la Real Universidad de San Felipe (Santiago, 1928), I, p. 202. Hanisch Espíndola, Walter, La filosofía de don Juan Egaña, en Historia, 3 (1964), p. 171.

${ }^{5}$ Guzmán Brito, Alejandro, cit. (n. 1), p. 13. Hace referencia a Lira MontT, Luis, Los colegios reales de Santiago de Chile, Reseña histórica e indice de colegiales. 1584-1816 (Santiago, 1977), p. 85. La beca era otorgada por los señores de este apellido. Asimismo, EncinA, Francisco Antonio, cit. (n. 3), I, p. 138, transcribe que la constancia de esta beca se encontraba "a fs. 121 del libro de asientos del antiguo consistorio de San Carlos", habiendo sido presentado por el Marqués de Casa Larraín. 
(las Institutiones de Justiniano, comentadas por el jurisconsulto holandés Arnoldo Vinnius $)^{6}$ las empezó a estudiar por medio de docencia privada impartida por su condiscípulo José Gabriel Palma Villanueva ${ }^{7}$, quedando constancia solo de haber revisado el primer libro durante el año $1815^{8}$.

El siempre crítico José Victorino Lastarria buscó dejar constancia de los aspectos más mundanos de su paso por este colegio: “se distinguió, más que por sus talentos, por un carácter dominante, travieso y arrojado, que auguraba en él más al revolucionario, que al hombre de letras y de estudio. Portales no aprendía nada en el colegio, pero subvertía el orden a cada paso e incomodaba a todos, tanto a los superiores como a sus compañeros, con picantes travesuras y extravagantes ocurrencias" ". Vicuña Mackenna también hace eco de estas travesuras juveniles: "Cuéntase de él que un día dio salida a todos los alumnos del establecimiento, quebrando las ollas de la cocina, a fin de que no hubiera alimento, y más de una ocasión vistió con las sotanas del rector (que regularmente era algún canónigo) su propia mula calesera" ${ }^{10}$.

Ese mismo año, el 1 de septiembre de 1808, y en cumplimiento de un decreto emanado por el rector Vicente Martínez de Aldunate de cuatro días antes ${ }^{11}$, Portales se matriculó, junto con otros nueve alumnos del Colegio Carolino y otros

${ }^{6}$ Una breve semblanza de Vinnius (o Vinnio) en Hanisch Espíndola, Hugo, Andrés Bello y su obra en derecho romano (Santiago, 1983), p. 44: "es el clásico comentarista de la ley, que se ciñe al texto y trata de explicarlo dentro de la técnica jurisprudencial, de una manera profunda, exhaustiva, pero con la oscuridad y densidad propia de su época y de la mentalidad formada dentro de la escolástica y la lógica deductiva más rigurosa". GonZáLEZ ECHEÑIQUE, Javier, Los estudios jurídicos y la abogacía en el reino de Chile (Santiago, 1954), p. 160, cita un testimonio del ex presidente Francisco Antonio Pinto para individualizar la obra específica estudiada como "In quatuor libros Institutionum Imperaialum comentarios academicus et forensis” (1642).

${ }^{7}$ Nacido en 1794 y graduado tanto en Teología (1813) como en Cánones y Leyes (bachiller, 1817; doctor, 1842), Palma Villanueva tendría una larga carrera posterior tanto como académico (tanto en la Universidad de San Felipe como en el Instituto Nacional y la Universidad de Chile), así como diputado (1843-1852) y por sobre todo, como ministro de la Corte Suprema (1852-1868). Murió el 3 de julio de 1881. FigueroA, Virgilio, Diccionario histórico y biográfico de Chile (Santiago, 1925-1931), IV-V, p. 462; Biblioteca del Congreso Nacional, José Gabriel Palma Villanueva. Reseñas biográficas parlamentarias [visible en internet: https://www.bcn.cl/historiapolitica/resenas_parlamentarias/wiki/Jos\%C3\%A9_Gabriel_Palma_Villanueva]

${ }^{8}$ Guzmán Brito, Alejandro, cit. (n. 1), pp. 13-14 y 16. Se remite igualmente a Vicuña Mackenna, Benjamín, cit. (n. 2), p. 24. Guzmán Brito explica lo anterior ya que Portales se encontraba en la necesidad de cursar primero el bachillerato en filosofía para luego optar al grado en derecho: "de guisa que resultaba inútil adelantar estudios jurídicos mientras cursaba los de filosofía". Véase nota 12.

${ }^{9}$ Lastarria, José Victorino, Don Diego Portales. Juicio bistórico (Santiago, 1861), ahora, en Lastarria, José Victorino; Vicuña Mackenna, Benjamín; Sotomayor Valdés, Ramón, Portales. Juicio histórico (Santiago, 1973), p. 13.

${ }^{10}$ Vicuña Mackenna, Benjamín, cit. (n. 2), p. 22. También mencionado por Encina, Francisco Antonio, cit. (n. 3), I, p. 138.

${ }^{11}$ Este decreto, así como sus antecesores, tienen su fundamento en uno de 18 de junio de 1794, firmado por el rector don Francisco Javier de Errázuriz, quien en pos de enmendar el desorden existente con las matrículas de leyes, "ordenó que en lo de adelante para poder ganar cursos debían los estudiantes, sin exceptuar a los religiosos o a los de estudios privados, de presentarse ante una comisión compuesta del Rector en persona, del secretario y del segundo 
20 estudiantes de otros orígenes ${ }^{12}$, para cursar estudios de filosofía en la Real Universidad de San Felipe, cuyo bachillerato era prerrequisito para poder estudiar el respectivo en cánones y leyes desde el año $1807^{13}$. Se matriculó nuevamente el 8 de mayo de 1810, cumpliendo con un nuevo decreto, esta vez del rector Juan José del Campo, y se sabe que obtuvo el primer grado el 18 de enero de $1811^{14}$, aunque se le contó igualmente como alumno en $1812 .{ }^{15}$

En ese tiempo, se desempeñaron como catedráticos cuatrianuales de Artes o Filosofía los siguientes académicos:

Francisco Semir (ganada en oposición el 23 de agosto de $1807^{16}$-declarada vacante el 3 de septiembre de 1810 por plazo cumplido-): se había graduado como bachiller en Teología el 22 de noviembre de 1796; de bachiller en Cánones y Leyes el 8 de febrero de 1798; como licenciado y doctor en Cánones y Leyes el 9 de octubre de 1803, y; finalmente de licenciado y doctor en Teología el 29 de agosto de 1808, todo por la misma Real Universidad de San Felipe ${ }^{17}$.

Fray Pedro Manuel Chaparro (24 de octubre de $1810^{18}$-declarada vacante el 3 de enero de 1812 por muerte-): de la Orden de San Juan de Dios, cuenta con una biografía bastante interesante: habiendo ingresado a estudiar circa 1767, en 1771 el presidente Francisco Javier de Morales, siendo rector Fernando Bravo de Nareda, ofreció dar informe sobre su solicitud de que se le concediera el grado de doctor en Medicina "a título de examinador, con respecto a la falta que de ellos había en la Facultad", graduándose de bachiller en tal materia el 27 de febrero de 1771 y de licenciado y doctor en ella con fecha 20 de julio de 1772, siendo "el primer estudiante universitario en Santiago que alcanzara tan alta distinción”. Medina indica que siguió estudios de teología, mas no perseveró en ellos. Sirvió largo tiempo como examinador, y asimismo fue el primero en experimentar el uso de la vacuna contra la viruela. Se presenta como opositor a la cátedra de Medicina en 21

consiliario mayor, a inscribirse con la precisa distinción de la Facultad cuyos cursos deseaban seguir”. Medina, José Toribio, cit. (n. 4), I, p. 361.

${ }^{12}$ Diez del Seminario (José Gabriel Palma, entre ellos), 2 dominicos, 10 de la orden de la Merced y 8 seculares de la misma orden. MedinA, José Toribio, cit. (n. 4), I, p. 564.

${ }^{13}$ Véase nota 7. Medina, José Toribio, cit. (n. 4), I, p. 313, transcribe: "Octavo, que en atención a que se ha multiplicado asombrosamente el estudio de la jurisprudencia, con abandono de las demás ciencias, sin duda por la facilidad de entrar en él sin otros principios, de que provienen no pocos inconvenientes: no se admitan en lo sucesivo al grado de bachiller en Leyes los que no lo bayan obtenido en Filosofía, y que el Secretario no matricule en adelante a persona alguna en la clase de Juristas sin que haga constar al señor rector haber estudiado Artes, entendiéndose lo mismo para Teología y Medicina; [...])".

${ }^{14}$ Medina, José Toribio, cit. (n. 4), I, p. 552: "1811. Enero 18. Filosofía. Diego Portales".

${ }^{15}$ Ibíd., I, p. 567.

${ }^{16}$ Se presentaron a esta oposición además fray Pedro Manuel Chaparro y los argentinos Bernardo Véliz, José de Amenábar y Antonio Álvarez de Jonte. Votaron 51 doctores: se sabe que 24 por Semir y 23 por Chaparro. El nombramiento del primero como docente no estuvo exento de polémica estuvo exento de polémica, puesto que éste "pretendía, en caso de admitirse a Chaparro, [este] debía dar fianzas de devolver los emolumentos que hubiera percibido, pues resultaba dudosa su admisión, según el texto de varias Reales disposiciones”. MedinA, José Toribio, cit. (n. 4), I, p. 412, n. 29.

${ }^{17}$ Ibíd., I, pp. 536, 538, 546-547 y 412.

${ }^{18}$ Medina, José Toribio, cit. (n. 4), t. 1, p. 506. 
de junio de 1779, en largo alegato contra don José Antonio de los Ríos; acogido este último, opone excepción de espurio, con alta polémica en la época, la cual fue desechada el 27 de mayo de 1782. Con fecha 6 de agosto de 1798, Chaparro es nombrado regente sin sueldo de la cátedra de Filosofía, "privando de ella a D. Ramón Arístegui, que cobraba la mitad del de planta", no sin resistencia de este último, que apelaría a una real cédula que impedía a los hospitalarios presentarse a oposiciones, alegación desestimada tanto en esta como en otras ocasiones. Se presentó a oposición nuevamente a esta cátedra el 22 de septiembre de 1803, obteniéndola; se mostró en rebeldía al tener que dejarla por fin del cuatrienio en mayo de 1807, abandonando incluso la ciudad. No obstante, el 23 de agosto de ese año postuló de nuevo, siendo derrotado por Francisco Semir, y; el 24 de octubre de 1810, preservándola esta vez hasta mayo de 1812, cuando por sus achaques no pudo desempeñarla más, falleciendo a fines de ese año ${ }^{19}$.

Manuel Verdugo, quien sirvió 8 meses como suplente por enfermedad y muerte de fray Chaparro, resolviéndose pagarle su salario con fecha 14 de enero de $1812^{20}$.

Portales se matriculó finalmente el 31 de agosto de 1812 para cursar el bachillerato en cánones y leyes; sin embargo, Guzmán Brito señala que "es seguro que no alcanzó a frecuentar ninguna clase de curso, dada la situación desarreglada en que por aquellos días se encontraba dicho establecimiento; tampoco pudo seguirlos en el Convictorio Carolino que había cesado de funcionar en 1812"21. Esta situación de desarreglo se manifestaba de distintas formas: ya en 3 de noviembre de 1810 el virrey propuso la supresión de la cátedra de Prima de Medicina ${ }^{22}$; la remoción y restitución del doctor Bernardo Vera y Pintado en su cátedra de Instituta en 1810-1811, por su detención en razón de la supuesta conspiración en su momento denunciada por el gobernador Francisco García Carrasco; ${ }^{23}$ el haberse notado "no sin grave dolor, la negligencia de los estudiantes en asistir a los cursos, llegando al extremo de que se queden muchos días sin actuación, porque los asignados a ella se ausentan o excusan con frívolos pretextos" ${ }^{24}$; el afán de don Manuel de Salas para fusionar la Academia de San Luis con el Colegio Carolino, pasándose a dictar las facultades de Filosofía, Leyes, Teología e incluso Matemáticas en este nuevo recinto ${ }^{25}$; la suspensión de la provisión de nuevas cátedras hasta nueva orden por oficio de la Junta de Gobierno de octubre de $1811^{26}$; la reducción de sueldos de catedráticos y el intento de suprimir la cátedra de Retórica a inicios de 1812, $\mathrm{y}^{27}$, finalmente, el traspaso de las labores docentes al nuevo y largamente

${ }^{19}$ Ibíd., (n. 4), I, pp. 151-153, 390- 506, 517, 530, 413-414.

${ }^{20}$ Ibíd.

${ }^{21}$ GuZmán Brito, Alejandro, cit. (n. 1), p. 13. Hace referencia a Lira Montt, Luis, cit. (n. 3), p. 27 y a Archivo Nacional, Real Universidad de San Felipe, vol. 7, pza. 3, fs. 264 v.

${ }^{22}$ Medina, José Toribio, cit. (n. 4), I, p. 330.

${ }^{23}$ Ibíd., cit. (n. 4), I, pp. 330 y 333.

${ }^{24}$ Ibíd., I, p. 335. Consta en informe previo para la erección de una cátedra de Derecho Natural y de Gentes.

${ }^{25}$ Ibíd., I, p. 336.

${ }^{26}$ Ibíd., I, p. 337. Se dio a conocer en sesión de 23 de ese mes y año.

${ }^{27}$ Ibíd., I, pp. 338-340. 
planeado Instituto $\mathrm{Nacional}^{28}$, decretado por el nuevo gobierno liderado por el brigadier José Miguel Carrera, quien también suspendió momentáneamente las cátedras de Matemáticas y Decreto ${ }^{29}$.

\section{Las Piezas inmediatas: Los estudios De don Diego Portales EN EL INSTITUTO NACIONAL}

Por lo anterior, y en vista que el nuevo Instituto Nacional inició funciones el día 10 de agosto de 1813, Portales se matriculó veinte días después en él, ${ }^{30}$ nuevamente bajo el amparo de la denominada "Beca Lecaros", como consta en la transcripción de su partida de matrícula: "Don Diego Portales, natural de esta ciudad, e hijo legítimo del señor superintendente de la Casa de Moneda don José Santiago Portales y de doña María Palazuelos, entró al Instituto, el día 30 de Agosto de 1813, a estudiar Derecho Naturaly de Gentes, es de diecinueve años, y ocupa la beca dotada de la familia de los señores Lecaros, por presentación que hizo de su persona el señor Márquez de Casa Larraín, según consta a fojas 121 del libro de asientos del antiguo Convictorio de San Carlos'\$1.

Considerando que el Instituto Nacional incluía dentro de los establecimientos previos fusionados al Seminario de Santiago, sus estudiantes ingresaban en dos categorías: seminaristas o pensionistas de carácter laico, más conocidos como convictoristas: en esta última categoría ingresaba Portales, así como el ya mencionado José Gabriel Palma, Fernando Elizalde ${ }^{32}$ y Juan Egaña hijo ${ }^{33}$, entre otros.

${ }^{28}$ Silva Castro, Raúl. Fundación del Instituto Nacional: 1810-1813 (Santiago, 1953), pp. 5-7 y 18 , señala que el Instituto Nacional como proyecto había sido concebido por don Juan Egaña en 1810, dejando incluso constancia de su afán en el proyecto inédito de Constitución que se le encargara por el Congreso Nacional en 1811. Fray Camilo Henríquez, por su parte, difundió en 18 y 25 de junio de 1812 un "Plan de Organización del Instituto Nacional de Chile", avalando la anterior idea. Finalmente, la redacción de las ordenanzas del Instituto recayó en quien fuera rector del desaparecido Colegio Carolino y que ocuparía idéntico cargo en el Instituto, don José Francisco de Echaurren, quien las presentó el 17 de julio de 1813 a la Junta de Educación.

${ }^{29}$ Medina, José Toribio, cit. (n. 4), I, p. 341.

${ }^{30}$ Guzmán Brito, Alejandro, cit. (n. 1), p. 15.

${ }^{31}$ Gajardo Villarroel, Enrique, Reseña histórica de la enseñanza superior en Chile y el estudio del derecho de gentes, antes y después de la independencia, en Revista Chilena, 12/97 (mayo de 1928), p. 460, señala que estas becas fueron creadas para favorecer a jóvenes de escasos recursos: en este caso, se entiende que los recursos de la familia Portales Palazuelos calificaban de ese modo en razón de estar conformada la prole por nada menos que veintitrés hermanos.

${ }^{32}$ Elizalde, graduado en Cánones y Leyes (1817) tuvo igualmente larga trayectoria tanto en el Poder Judicial (fiscal y luego ministro de la Corte de Apelaciones desde 1827, fiscal interino de la Corte Suprema en 1837) como en el Poder Ejecutivo (ministro interino de Hacienda dos veces en 1823) y Legislativo (diputado entre 1824-1825 y 1828-1830; senador entre 1831 y 1842). Falleció en 1842. Biblioteca del Congreso Nacional, Fernando Antonio Elizalde Marticorena. Reseñas biográficas parlamentarias [visible en internet: https://www.bcn.cl/historiapolitica/ resenas_parlamentarias/wiki/Fernando_Antonio_Elizalde_Marticorena]

${ }^{33}$ Nacido en 1799, recién se recibiría de abogado en 1832. Diputado (suplente 1837-1840, propietario 1846-1849). Intendente de Santiago (1848) y fiscal de la Corte de Apelaciones de Concepción. Opositor en la Revolución de 1851, fue desterrado a Juan Fernández tal como su padre en el pasado, escapándose en barco. En ese contexto adquirió graves problemas de salud. Falleció en 1871. FigueroA, Virgilio, cit. (n. 7), t. 3, p. 38. Biblioteca del Congreso Nacional, 
La cátedra de Derecho Natural y de Gentes se había puesto a cargo del presbítero don José María Argandoña ${ }^{34}$ : nacido en La Serena en 1788, se había graduado de bachiller en Filosofía el 23 de enero de 1808; del mismo grado en Teología, el 3 de febrero de 1809, y de licenciado y doctor en Cánones y Leyes recientemente, el 5 de diciembre de 1810. Había enseñado filosofía, economía política y derecho natural y de gentes en el Colegio Carolino, y se había presentado a la oposición a la cátedra de Instituta de la Real Universidad de San Felipe el 8 de octubre de 1812, perdiendo contra don Juan de Dios Arlegui. En el Instituto Nacional ejerció hasta la clausura del mismo por la toma de Santiago por el ejército realista de Mariano Osorio, el 17 de octubre de 1814. No existen mayores antecedentes sobre su situación en la Reconquista, pero se sabe que ya declarada la Independencia fue párroco de La Serena (1819-1821), racionero y canónigo doctoral de la Catedral de Santiago (1821-1823), diputado y senador suplente por Coquimbo (1823), protector de la libertad de imprenta (1823) y miembro de la comisión demarcadora de los departamentos del país (1823). Falleció en el ejercicio de estos últimos cargos en junio de $1823^{35}$.

En lo que respecta a los textos de estudio utilizados en esta cátedra, existe en esta materia plena claridad que se enseñaba el "Elementa juris naturae et gentium" ("Elementos del derecho natural y de gentes"), del año 1737, cuya autoría recaía en Johann Gottlieb Heinecke (latinizado Heineccius, traducido al castellano como Juan Teófilo Heinecio), jurista nacido en la ciudad de Eisemberg, ducado de Altenburgo (actual estado federado de Turingia, República Federal Alemana), el 11 de septiembre de 1681, y muerto en 31 de agosto de 1741 en la ciudad de Halle (actual estado federado de Sajonia-Anhalt, R.F.A.). Fue profesor de las Universidades de Halle (1708-1732), Franeker (1723-1727) y Frankfurt am Oder (1727), y se caracterizó por ser un continuador de Samuel Freirherr von Pufendorf, quien a su vez se encontraba inspirado por Hugo Grocio. ${ }^{36}$ Hugo Hanisch Espíndola nos hace una caracterización bastante precisa del sello jurídico de Heineccius: "Heinecio

Juan Maria Egaña Fabres. Reseñas biográficas parlamentarias [visible en internet: https://www.bcn. $\mathrm{cl} /$ historiapolitica/resenas_parlamentarias/wiki/Juan_Mar $\%$ C3\%ADa_Ega $\%$ C3\%B1a_Fabres]

${ }^{34}$ Gajardo Villarroel, Enrique, cit., (n. 31), p. 460, señala que "El hecho de que fuera un eclesiástico el primer catedrático de este ramo se explica porque él se estudiaba en la sección destinada a los que debían abrazar esa carrera, o sea, en el Seminario. Cosa curiosa: él no figuró en un principio en los estudios de jurisprudencia sino mucho más tarde".

${ }^{35}$ Guzmán Brito, Alejandro, cit. (n. 1), p. 15; Medina, José Toribio, cit. (n. 4), I, pp. 539 y 551; Figueroa, Virgilio, cit. (n. 7), t. 1, p. 570; Biblioteca del Congreso Nacional, José María Argandoña. Reseñas biográficas parlamentarias [visible en internet: https://www.bcn.cl/historiapolitica/resenas_parlamentarias/wiki/Jos\%C3\%A9_Mar\%C3\%ADa_Argando $\% \mathrm{C} 3 \% \mathrm{~B} 1 \mathrm{a}]$

${ }^{36}$ Pérez Godoy, Fernando, Johannes Heineccius y la historia transatlántica del ius gentium, en Revista Chilena de Derecho, 44/2 (2017), p. 540. Disponible en https://scielo.conicyt.cl/scielo. php?script=sci_arttext\&pid=S0718-34372017000200539; GAJARDO VILLARROEL, Enrique, cit., (n. 31), p. 458. Pufendorf, nacido en Chemnitz en 1632 y muerto en Berlín en 1694, es reconocido como "uno de los tratadistas más importantes de la Escuela Racionalista de Derecho Natural alemana, junto con Christian Wolff y Tomasius. Esto se debe a la capacidad que tuvo de proponer una antropología numerosa que conjugaba elementos del pensamiento de Grocio y Thomas Hobbes. Su punto de partida es la sociabilidad del hombre". Soberanes Fernández, José Luis. Sobre el origen de las declaraciones de derechos humanos (Ciudad de México, 2009), p. 107 [vi- 
no produjo una obra radical, ni dejó discípulos. Perteneció al grupo de juristas historicistas. El método de Heinecio obedecía a una concepción lógica, simple, eliminando toda discusión oscura y procurando sintetizar los conceptos básicos de los jurisconsultos romanos en axiomas, construidos a modo de mosaicos, integrados por los fragmentos precisos para exponer el pensamiento que pretendía, eliminando toda duda, obviando las disensiones, eliminando todo vestigio de discusión y dando la impresión que la obra jurisprudencial era un código sobrio, sistemático, definitivo, depurado y sistémico. Las modalidades germánicas y el ius hodiernum (derecho actual) son apéndices brevísimos al final de cada título resumidos en la misma forma que en el texto romano. El esquema de Heinecio era atractivo, lógico, seducía por la nítida presentación de las reglas y su eliminación de los casos y problemas menudos" ${ }^{37}$.

En términos más concretos: "Heinecio concibe el Derecho Natural de modo distinto al jusnaturalismo escolástico, considerando a la ley natural como producto de una reflexión racional dada a todos en común por Dios y no como una norma objetiva de orden ontológico inscrita por Él en la naturaleza. De este modo, de manera sutil pero real, el Derecho Natural de corte racionalista introduce la ruptura entre razón y naturaleza y proclama la primacía del primero sobre el segundo, iniciando de esta manera el camino para el positivismo jurídico que se enseñará a partir del siglo XIX" ${ }^{38}$.

La incorporación del texto de Heineccius en la enseñanza jurídica chilena podría ser considerada en Chile como un evento revolucionario, enmarcada en los cambios que a nivel educativo estaban transcurriendo ya hacía décadas en los focos más avanzados del Imperio, que buscaban desplazar el tradicional enfoque basado en la escolástica, para la instalación de un verdadero estudio iusnaturalista ${ }^{39}$, enmarcado dentro de las ideas de la ilustración católica y nacional a la que tantas veces se ha referido el profesor Bravo Lira ${ }^{40}$ : claro ejemplo de lo anterior es que, al fundarse el Convictorio de San Carlos en Lima, en el año 1771, las obras de Heineccius pasaron a ser la columna vertebral de los estudios: su "Historia de la Filosofía" era parte del primer año del curso de filosofía, mientras que en Jurisprudencia, en primer año se revisaba el ya mencionado "Elementos del Derecho naturaly de gentes", mientras que al segundo, correspondía a las "Instituciones

sible en internet: https://archivos.juridicas.unam.mx/www/bjv/libros/6/2698/12.pdf] [Libro completo visible en internet: http://ru.juridicas.unam.mx/xmlui/handle/123456789/11608]

${ }^{37}$ Hanisch Espíndola, Hugo, cit. (n. 6), p. 43.

${ }^{38}$ Valle Rondón, Fernando. Teología, filosofía y derecho en el Perú del XV III: dos reformas ilustradas en el Colegio de San Carlos de Lima (1771 y 1787), en Revista Teológica Limense, 40/3 (2006), p. 343 [visible en internet: http://textos.pucp.edu.pe/pdf/2086.pdf]

${ }^{39}$ Pérez Godoy, Fernando, cit. (n. 36), p. 545.

${ }^{40} \mathrm{El}$ autor citado ha caracterizado a la Ilustración "católica y nacional" por medio de la obra de sus principales exponentes. En ese sentido, véase BrAvo LiRA, Bernardino, Feijoo y la Ilustración católica y nacional en el mundo de habla castellana y portuguesa, en Jabrbuch für Geschichte Lateinamerikas, 22 (1985), pp. 99-122; ÉL MISMO, Jovellanos y la Ilustración católica y nacional en el mundo de habla castellana y portugués, en Revista de Estudios Histórico-Jurídicos, 9 (1984), pp. 113-166; ÉL MISMO, Melo Freire y la ilustración católica y nacional en el mundo del habla castellana y portuguesa, en Revista de Derecho de la Pontificia Universidad Católica de Valparaíso, 8 (1984), pp. 72-128; entre otros. 
de Justiniano" y en el tercero, el "Tratado o Libro de las Antiguedades Romanas" ". Esta influencia se vio incluso potenciada durante el fecundo período como rector de Toribio Rodríguez de Mendoza (1786-1817), eximio pedagogo adelantado a su tiempo, quien reafirmando la presencia predominante de este autor en el plan de estudios de 1787, "consideraba que en los estudios jurídicos se debía privilegiar la enseñanza del Derecho natural, español, civil y canónico. Cabe resaltar la incorporación del derecho natural de corte racionalista como nueva disciplina y el derecho español en los planes de estudios" ${ }^{42}$.

Esta influencia determinante y creciente en el tiempo no podía sino contrastar con el hecho que Heineccius había sido, en los hechos, incorporado en el Índex de Libros prohibidos tanto por la Sacra Congregación Romana y por la Santa Inquisición Española en tiempos no muy lejanos (1743 y 1745, nada menos), debiéndose usar para estos eventos una edición previamente censurada por Joaquín Marín y Mendoza (1727-1782), primer profesor de derecho natural de España, legitimada por el poder central hispánico desde $1776^{43}$.

¿Cómo llegó, entonces, la influencia de Heineccius a Chile? El personaje clave en este punto es el destacado jurista Juan Egaña Risco (1769-1836). Si bien él realizó sus primeros estudios en el Colegio Seminario de Santo Toribio de Mogrovejo, este último recinto no se vio al margen del espíritu reformista ni de la enseñanza de Heineccius: el panameño Agustín de Gorrochatégui en su rectorado (17601770), será el encargado de introducir la teología positiva y el estudio directo de las fuentes bíblicas y conciliares ${ }^{44}$, lo cual sería consolidado bajo la regencia del eclesiástico Baltazar Martínez y Compañón (1770-1778), quien posteriormente fuera obispo de Trujillo, pues en este período se efectuó una reforma de estudios bajo la supervisión del mencionado Rodríguez de Mendoza ${ }^{45}$. Si bien desde su llegada a Chile su labor académica, aparte del ejercicio profesional, estuvo principalmente centrada en la retórica y en el estudio de la prosa y poesía latinas (habiendo sido nombrado consiliario menor de la Real Universidad de San Felipe el 13 de diciembre de 1791 y catedrático de Latinidad y Retórica desde el 5 de agosto de 1802, cátedra que él mismo contribuyó a fundar), mostrándose recurrentemente crítico sobre el estado de los estudios de jurisprudencia en el país ${ }^{46}$, fue recién

\footnotetext{
${ }^{41}$ Huaraj Acuña, Juan Carlos, El Convictorio de San Carlos de Lima. Curriculo y pensamiento educativo, 1771-1836. (Lima, 2007), p. 77.

${ }^{42}$ SÁnchez Raygada, Carlos Hugo. La propuesta educativa de Toribio Rodríguez. de Mendoza y la enseñanza jurídica en el Convictorio de San Carlos de Lima, en YANZI FerReira, Ramón Pedro (coord.), XVIII Congreso Instituto Internacional de Historia del Derecho Indiano (Córdoba, 2016), pp. 762 y ss.

${ }^{43}$ Pérez Godoy, Fernando, cit. (n. 36), pp. 544-547; Valle Rondón, Fernando, cit. (n. 38), pp. 544-547. Sobre la adaptación de Heinecio a la dogmática católica, véase Pérez GodoY, Fernando, La teoría del derecho natural y de gentes de Johannes Heineccius en la cultura jurídica iberoamericana, en Revista de Estudios Histórico-Jurídicos, 37 (2015), pp. 453-474; en especial, pp. 459-464. [visible en internet: https://scielo.conicyt.cl/pdf/rehj/n37/a17.pdf]

${ }^{44}$ Perú Cristiano, El blog de la Historia de la Iglesia Católica del Perú. "Martínez de Compañón, rector del Seminario de Santo Toribio de Lima de 1770 a 1778". Publicado el 2 de septiembre de 2018. [visible en internet: http://peru-cristiano.blogspot.com/2018/09/martinez-de-companonrector-del.html]

${ }^{45}$ Valle Rondón, Fernando, cit. (n. 38), pp. 545.

${ }^{46}$ Silva Castro, Raúl. Egaña en la Patria Vieja (Santiago, Editorial Andrés Bello, 1958), pp. $20-24$
} 
ante vientos más favorables a los cambios, desde 1810 en adelante, en que pudo manifestar en plenitud su interés por una reforma educacional conforme a los cánones ilustrados: ya en agosto de ese año presentó al Conde de la Conquista la idea que "la obra de Chile debe ser un gran colegio de artes y ciencias, [capaz de impartir] una educación civil y moral capaz de darnos costumbres y carácter" ${ }^{47}$.

Este anhelo, que implicaría la fusión de todos los planteles educativos existentes, cada cual con una existencia más lánguida respecto del otro, fue respaldado primero por fray Camilo Henríquez en su "Plan de Organización del Instituto Nacional de Chile", ${ }^{48}$ y luego tanto por la Junta de Gobierno como por el Senado, con la única oposición del Seminario de Santiago, situación que se zanjó finalmente con el pacto de un concordato entre el representante del gobierno (el mismo Juan Egaña, nombrado el 29 de junio de 1813) y el del obispado (el párroco de Talca, Juan Ignacio Cienfuegos, nominado a su vez por el regente provisional Rafael Andreu y Guerrero), firmado el 25 de julio de 1813, mientras que en simultáneo se daban a la luz las Ordenanzas del Instituto, redactadas por José Francisco de Echaurren y con adiciones del mismo Egaña ${ }^{49}$. Respecto a la constitución de su planta de cátedras (bastante heterogénea, vista la diversidad de establecimientos que concurrieron a su formación) y de los contenidos que en éstas se pasarían, la Junta de Educación (conformada por los mismos Egaña y Echaurren) pidió una serie de informes para asesorarse en ese sentido; es innegable la injerencia del primero en este sentido a la hora de determinar cómo se encauzarían los estudios de derecho en la nueva institución. Y las ordenzanzas terminaron siendo bastante explícitas en ese sentido, tanto en sus afanes: "Elpublicista aprenderá en las lecciones del derecho natural, de jentes i economía politica, los medios de establecer, conservar $i$ mejorar las relaciones del pais con justicia i provecho. No nadará incierto entre la opinion $i$ el riesgo. Estudiará en la historia los avisos de todos los tiempos, i leerá en los consectarios de los primeros principios los de la virtud, que debe conducirlo, $i$ los infelices del vicio, de la intriga $i$ maquiavelismo, que ha de detestar. Esta cátedra sitúa el instituto. El majistrado, despues de formar su corazon por los principios de la sana moral, aprenderá a manejar, por la ética, los resortes maestros del alma en sus semejantes. Se hará liberal, accesible i bumano, por la buena filosofía, i un profesor profundo del derecho, por el estudio del natural, civil, canónico i patrio, que dictará la matriz jeneral" 50 .

Como al determinar los contenidos en sí mismos: "Sin profesores del derecho no hai buena administracion, ni pueden formarse aquéllos sin las lecciones del natural que, aunque cincelado sobre el corazon del hombre, tiene leyes precisas e inmutables, de donde se derivan las de jentes i patrias. Estas deducciones, para ser exactas, suponen el conocimiento de los resortes del corazon, que enseña la ética ofilosofía natural; i los deberes i derechos del hombre en sociedad, que dicta la economía política. Por eso se establece la cátedra del derecho natural i de jentes, que ha de dictarse por Heineccio; con lecciones de economía politica por Genovesi, Sunid i Say; i absolverá su curso en dos años (31).

${ }^{47}$ Citado por Silva Castro, Raúl, Fundación, cit. (n. 28), p. 5.

${ }^{48}$ La Aurora de Chile, 18 y 25 de junio de 1812.

${ }^{49}$ Silva Castro, Raúl, Fundación, cit. (n. 28), pp. 7-11.

${ }^{50}$ Acta de los acuerdos de la Junta de Gobierno, el Senado i el Cabildo de Santiago, en 27 de julio de 1813, en Letelier, Valentín, Sesiones de los Cuerpos Lejislativos de la República de Chile (1810-1845), I: 1810-1814 (Santiago, Imprenta Cervantes, 1887), pp. 289-322, en particular p. 297. 
Asi preparado el jurisconsulto, i prévio un tratado bistórico compendiado de las épocas, majistraturas i progresos del derecho romano, estudiará brevemente las instituciones de Justiniano (32), en su letra, con solo la esposicion de: catedrático, que ha de absolver en seis meses. Continuará el derecho real $i$ canónico: el primero por la instituta de Castilla, i el compendio de las leyes de partida por Viscaino, i el segundo, por el obispo Ananiense o el Selbagio, para lo que se sitúa la cátedra de derecho civil, real i canónico, que ha de absolver cada curso en dos años (33).

Bajo de estos principios pasará el abogado a la práctica, en que solo tendrá que aprender el órden de los juicios i las fórmulas del Toro, a que es mui bastante un año. I a él quedara reducida la práctica del tribunal, sirviéndole para el completo de los cuatro antes prevenidos, los empleados tambien, en parte del estudio del derecho natural, ética i economía política, $i$ en el de derecho civil, patrio i canónico. Los actuales practicantes no serán recibidos al ejercicio forense sin cursar i ser aprobados en estos estudios" ${ }^{51}$.

En este punto, como bien se puede observar, retomamos el camino original de nuestra investigación: la fundación del Instituto Nacional por acuerdo de Junta de Corporaciones (Junta de Gobierno, Senado y Cabildo de Santiago) dos días después de la firma del concordato y su puesta en funciones el 10 de agosto de ese año, con Echaurren como primer rector $^{52}$.

\section{LA PIEZA FINAL: DeSCRIPCIÓN DEL DOCUMENTO DE INTERÉS}

El documento de interés se transcribe del siguiente modo:

"Don Diego Portales, convictorista.

En 18 de Abril de 1814, el alumno convictorista Don Diego Portales fue examinado en la primera parte del Primer libro de Derecho Natural del jurisconsulto Heinnecio por el Sr. Rector Dr. Don Francisco de Echaurren, y catedráticos Dr. Don Juan de Dios Arlegui y Dr. Don José María Argandoña, y don Pedro Nolasco Carvallo, y salió aprobado, nemine discrepante, de que doy fe.

Firma: Juan de Dios Arlegu?".

Analizando el detalle del documento, puede observarse lo siguiente:

1. La comisión se encontraba presidida por el Rector del Instituto, don Juan Francisco de Echaurren, mientras que los demás miembros eran precisamente aquellos catedráticos vinculados directamente a la enseñanza jurídica: por Derecho Civil, Canónico y Leyes Patrias, el presbítero Juan de Dios Arlegui, quien a la vez hace de ministro de fe de la Comisión; de Derecho Natural y de Gentes, Economía Política y Filosofía Moral, el presbítero José María Argandoña, ya mencionada su semblanza en este documento, y; por Lógica y Metafísica, don Pedro Nolasco Carvallo ${ }^{53}$.

2. Del catedrático Juan de Dios Arlegui Rodríguez no existen mayores referen-

\footnotetext{
${ }^{51}$ Ibíd., p. 304. El destacado es nuestro.

${ }^{52}$ LeTELIER, Valentín, cit. (n. 50), Actualmente el profesor Antonio Dougnac se encuentra estudiando este tema en detalle, encontrándose su investigación aún inédita.

${ }^{53}$ Silva Castro, Raúl, Fundación, cit. (n. 28), p. 20.
} 
cias que las otorgadas por Virgilio Figueroa ${ }^{54}$ : fue calificado muy elogiosamente por Bernardo Vera y Pintado cuando este era presidente de la Academia de Práctica Forense (1804), y antes de ser presbítero, se desempeñó como abogado y además había ejercido previamente en el Convictorio Carolino las cátedras de Prima de Cánones, Instituta y Pasante; en el Instituto Nacional, aparte de la cátedra aquí mencionada, posteriormente ocupó la de Derecho Natural y de Gentes. Ya consagrado, fue secretario de su tío, el obispo Rodríguez Zorrilla, siguiendo su destino en los constantes destierros y relegaciones que este sufrió. Finalmente, fue desterrado en 1825 y falleció de vó m i to n e gro en alta mar, cruzando el Golfo de México. Por su parte, del catedrático Pedro Nolasco Carvallo Noriega no se encontraron antecedentes consistentes, más allá de algunas referencias genealógicas ${ }^{55}$.

3. Sobre los contenidos específicamente evaluados, si bien en estos momentos no hay certeza de cuál fue la edición específica de los Elementos del Derecho Natural $y$ de Gentes usada para su evaluación, es posible obtener una perspectiva gracias a ediciones posteriores, traducidas al castellano. Si tomamos como referencia la edición de 1837 de este texto ${ }^{56}$, el Libro I (o primera parte) sobre Derecho Natural, tiene un total de quince capítulos, los cuales se titulan del siguiente modo: Capítulo I: De la naturaleza y constitución del Derecho Natural y de Gentes; Capítulo II: De la naturaleza e índole de las acciones humanas; Capítulo III: De la norma de las acciones humanas y verdadero principio del derecho natural; Capítulo IV: De la aplicación a esta norma á las acciones, y diferencia de éstas que de aquí se originan; Capítulo V: De los deberes del hombre para con Dios; Capítulo VI: Deberes del hombre para consigo mismo; Capítulo VII: De los deberes absolutos y perfectos para con los demás hombres, especialmente el de no dañar a nadie; Capítulo VIII: De los deberes imperfectos para con los demás; Capítulo IX: De los deberes hipotéticos para con otros, y en primer lugar acerca de la adquisición originaria del dominio; Capítulo X: De las adquisiciones derivativas del dominio que se ejecutan viviendo su anterior dueño; Capítulo XI; De las adquisiciones derivativas por sucesión, disposición del difunto ó abintestato; Capítulo XII: De los derechos y deberes que se originan del dominio; Capítulo XIII: Del comercio de las cosas que están sujetas al dominio; Capítulo XIV: Sobre los pactos; Capítulo $\mathrm{XV}$ : Por qué medios se deshacen las obligaciones dimanadas de los contratos.

El esquema anterior nos permite tener un punto de partida inicial para considerar cuáles son los contenidos que fueron evaluados en esta oportunidad, que

${ }^{54}$ FigueroA, Virgilio, cit. (n. 7), I, pp. 593-594.

${ }^{55}$ Pilleux Cepeda, Mauricio. Genealogía de la Familia Carvallo (2021) [visible en internet: https://www.genealog.cl/Sur/C/Carvallo/]. Aparece también mencionado en PIÑERA, Bernardino. Piñera Carvallo. Historia de una familia (s.f.) [visible en internet: http://www.aninon.cl/ bernard/libros/historiadeunaFamilia.pdf] p. 63, documento relativo, entre otros, a la parentela del destacado diplomático José Piñera Carvallo y de su infausta descendencia, que debido a su mala fama haremos respecto de ella prudente "damnatio memoriae" en estas líneas.

${ }^{56}$ Heineccius, Johann Gottlieb, Elementos del derecho natural y de gentes de Heinecio, corregidos y reformados por el profesor don Mariano Lucas Garrido, á los que añadio los de la filosofía moral del mismo autor, y traducidos al castellano por el bachiller en leyes D. J. A. Ojea, Tomo I (Madrid: Imprenta que fue de Fuentenebro, a cargo de Alejandro Gómez, 1837). 
esencialmente se enmarcan en tres puntos esenciales: (1) nociones básicas de derecho natural (caps. I-IV); (2) esquema de deberes a los que está sometida la persona (caps. V-IX) y; (3) nociones básicas de derecho privado, particularmente sobre propiedad y actos jurídicos (caps. X-XV).

4. Asimismo, la expresión "nemine discrepante" quiere decir, literalmente, "sin que nadie disienta": la aprobación de Portales de este examen fue unánime, lo cual permite deducir la calidad de la presentación del futuro Ministro.

\section{Colofón: El CUADRo ÍNTEGRO}

Las conclusiones ante lo expuesto no pueden sino ser modestas, tanto por lo acotado del tópico tratado como por ya haber sido tratado el tema global en que éste se introduce con mayor detalle y claridad, de la cual se asume como complemento desde el primer momento. Por lo anterior, este documento siempre será recomendable que se lea como anexo a la obra referenciada del profesor Alejandro Guzmán Brito.

De todos modos es posible decir, incorporada esta pieza faltante del rompecabezas, que la formación intelectual del futuro ministro Portales, si bien debe ser caracterizada como irregular inevitablemente visto el contexto histórico en que se desenvuelve, en caso alguno podría decirse que esta fue insuficiente; todo lo contrario, en ella se ven los sustratos intelectuales prácticos e ilustrados que caracterizaron su obra y que, en los hechos, la volvieron permanente: una idea basal de equidad práctica y sentido de orden y justicia, tan necesarios en su tiempo y tan determinantes finalmente para nuestra historia republicana.

Diego Portales fue un hombre de su tiempo y por ende, debía ser más un hombre de acción que de reflexión, pero no podríamos decir que su genio e instinto hubieran llegado a la maestría que le caracterizó de no ser, precisamente, por esta formación jurídica basal, que hoy ya podemos decir, ya está completamente rastreada. El puzle está, por fin, completo. 


\section{ANEXo FotográFICO}

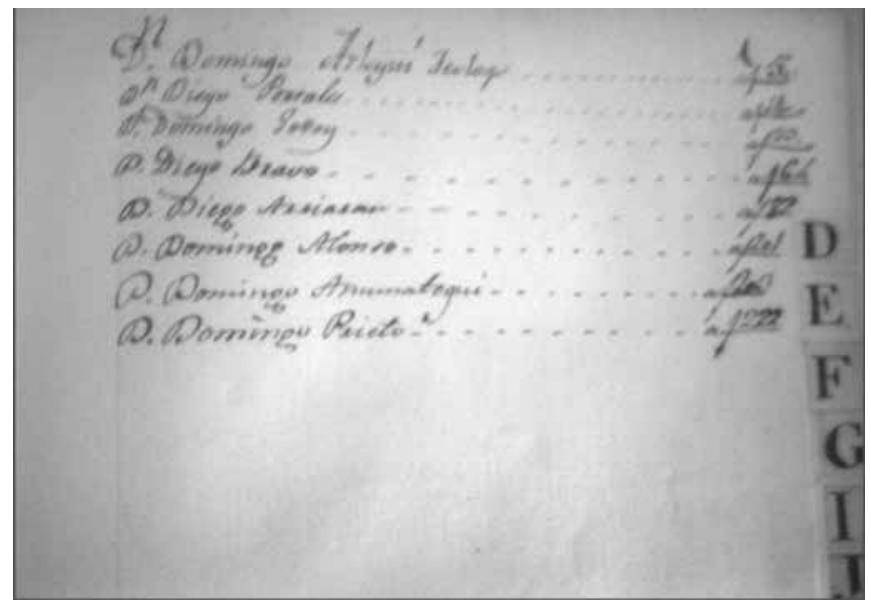

Fotografía tomada del índice del Libro de Exámenes del Instituto Nacional (1813-1822), tomo A-1, año 1814. Allí consta el nombre de don Diego Portales. Su baja resolución es por haber sido tomada con un teléfono celular de la época.

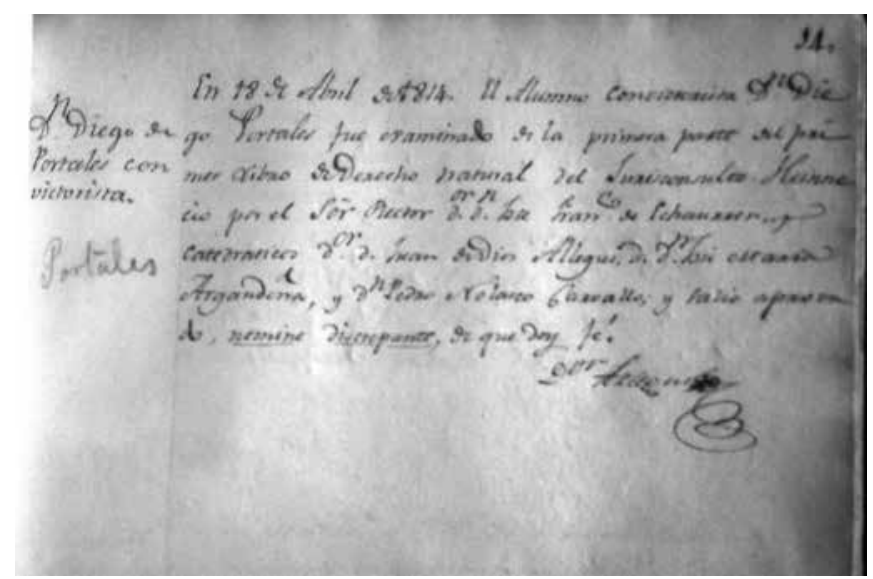

Fotografía tomada del Libro de Exámenes del Instituto Nacional (1813-1822), tomo A-1, año 1814, página 14, donde consta el examen de Derecho Natural rendido por don Diego Portales. Su baja resolución es por haber sido tomada con un teléfono celular de la época. 
Biblioteca del Congreso Nacional, José María Argandoña. Reseñas biográficas parlamentarias [visible en internet: https://www.bcn.cl/historiapolitica/resenas_parlamentarias/wiki/Jos\%C3\%A9_Mar\%C3\%ADa_Argando\%C3\%B1a]

—Fernando Antonio Elizalde Marticorena. Reseñas biográficas parlamentarias [visible en internet: https://www.bcn.cl/historiapolitica/resenas_parlamentarias/wiki/ Fernando_Antonio_Elizalde_Marticorena]

_-Juan María Egaña Fabres. Reseñas biográficas parlamentarias [visible en internet: https:// www.bcn.cl/historiapolitica/resenas_parlamentarias/wiki/Juan_Mar\%CC3\%ADa_ Ega\%C3\%B1a_Fabres]

Bravo Lira, Bernardino, Feijoo y la Ilustración Católica y Nacional en el mundo de babla castellana y portuguesa, en Jabrbuch für Geschichte Lateinamerikas, 22 (1985), pp. 99-122.

- Jovellanos y la Ilustración católica y nacional en el mundo de habla castellana y portugués, en Revista de Estudios Histórico-Jurídicos, 9 (1984), pp. 113-166.

- Melo Freire y la ilustración católica y nacional en el mundo del habla castellana y portuguesa, en Revista de Derecho de la Pontificia Universidad Católica de Valparaíso, 8 (1984), pp. 72-128.

Encina, Francisco Antonio, Portales. Introducción a la historia de la época de don Diego Portales. (Santiago, Editorial Nascimento, 1934), 2 vols.

Figueroa, Virgilio, Diccionario histórico y biográfico de Chile (Santiago, Imprenta y Litografía La Ilustración, 1925-1931), 5 vols.

Gajardo Villarroel, Enrique, Reseña histórica de la enseñanza superior en Chile y el estudio del derecho de gentes, antes y después de la independencia, en Revista Chilena 12/97 (mayo de 1928), pp. 448-493. Se realizó asimismo tirada especial de 1.000 ejemplares (Santiago, Dirección General de Talleres Fiscales de Prisiones, Taller Imprenta, 1928) [visible en internet: https://obtienearchivo.bcn.cl/obtienearchivo?id=doc umentos/10221.1/36312/1/215205.pdf]

GonzÁLEZ ECHEÑIQue, Javier, Los estudios jurídicos y la abogacía en el reino de Chile (Santiago, Facultad de Ciencias Jurídicas, Políticas y Sociales Universidad Católica de Chile, 1954).

Guzmán Brito, Alejandro, Las ideas jurídicas de Portales, en Boletin de la Academia Chilena de la Historia, 49/93 (1982), pp. 13-42; ahora, en ÉL MISMO, Portales y el derecho (Santiago, Editorial Universitaria, 1988), pp. 12-50.

Hanisch Espíndola, Hugo, Andrés Bello y su obra en derecho romano (Santiago, Ediciones del Consejo de Rectores de las Universidades Chilenas, 1983).

Hanisch Espíndola, Walter, La filosofía de don Juan Egaña, en Historia, 3 (1964), pp. 164-310.

HeIneccius, Johann Gottlieb, Elementos del derecho naturaly de gentes de Heinecio, corregidos y reformados por el profesor don Mariano Lucas Garrido, á los que añadió los de la filosofía moral del mismo autor, y traducidos al castellano por el bachiller en leyes D. J. A. Ojea. Tomo I (Madrid: Imprenta que fue de Fuentenebro, a cargo de Alejandro Gómez, 1837).

Huaraj Acuña, Juan Carlos El Convictorio de San Carlos de Lima. Currículo y pensamiento educativo, 1771-1836 (Tesis para optar el título de Licenciado en Historia, Universidad Nacional Mayor de San Marcos, Facultad de Ciencias Sociales, Escuela de Académico Profesional de Historia, Lima, 2007).

Lastarria, José Victorino, Don Diego Portales. Juicio histórico, (Santiago, Imprenta del Correo, 1861), ahora, en Lastarria, José Victorino; Vicuña Mackenna, Benja- 
mín; Sotomayor Valdés, Ramón, Portales. Juicio histórico (Santiago, Editorial del Pacífico, 1973), pp. 11-81.

Letelier, Valentín. Sesiones de los Cuerpos Lejislativos de la República de Chile (1810-1845) (Santiago, Imprenta Cervantes, 1887-1908), 37 vols.

Lira Montt, Luis. Los colegios reales de Santiago de Chile, Reseña histórica e indice de colegiales. 1584-1816 (Santiago, Academia Chilena de la Historia, 1977).

Medina, José Toribio, Historia de la Real Universidad de San Felipe (Santiago, Imprenta y Litografía Universo, 1928), 2 vols.

Pérez Godoy, Fernando, Johannes Heineccius y la historia transatlántica del ius gentium, en Revista Cbilena de Derecho, 44/2 (2017), pp. 539-562 [visible en internet: https:// scielo.conicyt.cl/scielo.php?script=sci_arttext\&pid=S0718-34372017000200539].

—La teoría del derecho naturaly de gentes de Johannes Heineccius en la cultura jurídica iberoamericana, en Revista de Estudios Histórico-Jurídicos, 37 (2015), pp. 453-474 [visible en internet: https://scielo.conicyt.cl/pdf/rehj/n37/a17.pdf]

Perú Cristiano. El blog de la Historia de la Iglesia Católica del Perú. "Martínez de Compañón, rector del Seminario de Santo Toribio de Lima de 1770 a 1778". Publicado el 2 de septiembre de 2018 [visible en internet: http://peru-cristiano.blogspot.com/2018/09/ martinez-de-companon-rector-del.html]

PIÑERA, Bernardino. Piñera Carvallo. Historia de una familia (s.f.) [visible en internet: http://www.aninon.cl/bernard/libros/historiadeunaFamilia.pdf]

Pilleux Cepeda, Mauricio, Genealogía de la familia Carvallo (2021) [visible en internet: https://www.genealog.cl/Sur/C/Carvallo/].

SÁnchez RAYgada, Carlos Hugo, La propuesta educativa de Toribio Rodríguez de Mendoz̨a y la enseñanza jurídica en el Convictorio de San Carlos de Lima, en Yanzi Ferreira, Ramón Pedro (coord.), XVIII Congreso Instituto Internacional de Historia del Derecho Indiano (Córdoba, Argentina, Instituto Internacional de Historia del Derecho Indiano, Facultad de Derecho y Ciencias Sociales de la Universidad Nacional de Córdoba y Facultad de Derecho y Ciencias Sociales de la Universidad Católica de Córdoba, 2016), pp. 749-772.

Silva Castro, Raúl. Egaña en la Patria Vieja (Santiago, Editorial Andrés Bello, 1958). —Fundación del Instituto Nacional: 1810-1813 (Santiago, Editorial Universitaria, 1953). Soberanes Fernández, José Luis. Sobre el origen de las declaraciones de derechos bumanos (Ciudad de México, Instituto de Investigaciones Jurídicas UNAM. Comisión Nacional de los Derechos Humanos, México, 2009). [Capítulo citado visible en internet: https://archivos.juridicas.unam.mx/www/bjv/libros/6/2698/12. pdf] [Libro completo visible en internet: http://ru.juridicas.unam.mx/xmlui/ handle/123456789/11608]

Valle Rondón, Fernando. Teología, filosofía y derecho en el Perú del XVIII: dos reformas ilustradas en el Colegio de San Carlos de Lima (1771 y 1787), en Revista Teológica Limense, 40/3 (2006), pp. 337-382 [visible en internet: http://textos.pucp.edu.pe/ pdf/2086.pdf]

Vicuña MaCKenna, Benjamín. Introducción a la historia de los diez años de la administración Montt. Don Diego Portales ${ }^{3}$ (Santiago, Editorial del Pacífico, 1974). 